\title{
Ceratopatia bolhosa: etiopatogênese e tratamento
}

\author{
Bullous keratopathy: etiopathogenesis and treatment
}

\author{
Eliana Doming'ues Gonçalves ${ }^{1}$ \\ Mauro Campos $^{2}$ \\ Fabiana Paris ${ }^{3}$ \\ José Álvaro Pereira Gomes ${ }^{4}$ \\ Charles Costa de Farias ${ }^{5}$
}

\begin{tabular}{|l|}
\hline RESUMO \\
\hline A ceratopatia bolhosa caracteriza-se pelo edema corneano estromal \\
acompanhado de bolhas epiteliais e subepiteliais devido à perda de \\
células e/ou alterações da junção endotelial. Nos casos mais avançados, \\
ocorre espessamento do estroma e presença de fibrose subepitelial e \\
vascularização corneana. Apresenta baixa de acuidade visual devido à \\
diminuição da transparência da córnea e pode estar acompanhada de \\
sensação de corpo estranho, lacrimejamento e dor devido as alterações \\
epiteliais como a presença de bolhas íntegras ou rotas. Ceratite bolhosa \\
é uma das principais causas de transplante de córnea em diferentes regiões \\
e países. A principal etiologia é a perda de células endoteliais, principal- \\
mente após cirurgia de catarata e na distrofia endotelial de Fuchs. Sabe- \\
se que atualmente há cerca de 20 milhões de pessoas com catarata no \\
mundo, e esta complicação pode afetar 1 a $2 \%$ das cirurgias de catarata. \\
Este texto faz uma revisão sobre a etiopatogênese da ceratopatia bolhosa \\
e sobre os tratamentos clínicos e cirúrgicos disponíveis para a doença. \\
\hline
\end{tabular}

Descritores: Doenças da córnea/terapia; Doenças da córnea/cirurgia; Transplante de córnea/etiologia; Extração de catarata
Trabalho realizado no Setor de Cirurgia Refrativa do Departamento de Oftalmologia da UNIFESP - São Paulo (SP) - Brasil.

${ }^{1}$ Pós-Graduanda do Departamento de Oftalmologia da Universidade Federal de São Paulo - UNIFESP - São Paulo (SP) - Brasil.

${ }^{2}$ Professor Livre Docente, Professor Chefe do Setor de Cirurgia Refrativa do Departamento de Oftalmologia da UNIFESP - São Paulo (SP) - Brasil.

${ }^{3}$ Pós-Graduanda do Departamento de Oftalmologia da UNIFESP - São Paulo (SP) - Brasil.

${ }^{4}$ Doutor, colaborador do Setor de Córnea e Doenças Externas do Departamento de Oftalmologia da UNIFESP - São Paulo (SP) - Brasil.

${ }^{5}$ Pós-Graduando do Departamento de Oftalmologia da UNIFESP - São Paulo (SP) - Brasil.

Endereço para correspondência: Eliana Domingues Gonçalves. Rua Borges Lagoa, 368 - São Paulo (SP) CEP 04038-002

E-mail: elianadgoncalves@terra.com.br

\section{INTRODUÇÃO}

A ceratopatia bolhosa (CB) caracteriza-se pelo edema corneano estromal acompanhado de bolhas epiteliais e subepiteliais devido à perda de células e/ou disfunção da função endotelial. Nos casos mais avançados, ocorre espessamento do estroma acima de $650 \mu$ e presença de fibrose subepitelial, podendo formar uma camada de colágeno retrolental ou membrana fibrótica retrolental e vascularização corneana.

Induz baixa de acuidade visual devido à diminuição da transparência e pode ser acompanhada de sensação de corpo estranho, lacrimejamento e dor devido às alterações epiteliais como a presença de bolhas íntegras ou $\operatorname{rotas}^{(1-6)}$.

Apesar destes conceitos serem bem estabelecidos, estudos imuno-histoquímicos mostram que ainda se tem muitas dúvidas em relação à etiopatogênese da CB. Os pacientes com CB com ou sem prognóstico de visão, sintomáticos, apresentam muitas limitações em suas atividades diárias, devido a dor, e fotofobia, mesmo apresentando acuidade visual boa no olho contralateral. Evitam a exposição ao sol, ficando limitados ao ambiente doméstico e muitas vezes apresentam insônia devido à dor. Estão sempre sinalizando seu desconforto intenso nas freqüentes visitas ao consultório. Existem várias propostas para o seu tratamento, que é inicialmente feito com uso de agentes hipertônicos, lentes de contato terapêuticas, e posteriormente com tratamentos cirúrgicos como punções estromais, membrana amniótica, ceratectomia lamelar e outros. Tratamentos 
para a melhora da dor enquanto o paciente espera o transplante de córnea, o qual pode demorar em muitos locais em torno de dois anos ou mais. A CB é uma das principais causas de transplante de córnea em diferentes regiões e países ${ }^{(3,6-13)}$.

O objetivo deste trabalho é de revisar os principais estudos relacionados à etiopatogênese da $\mathrm{CB}$ e os tratamentos possíveis para esta doença.

\section{Etiopatogênese}

A principal etiologia é a perda de células endoteliais pelo trauma cirúrgico, principalmente na cirurgia de catarata, na maioria dos casos acometendo pacientes acima da sexta década $^{(1,3)}$. Também ocorrem após cirurgias múltiplas para glaucoma, como trabeculectomia e tubo, retinopexias, fixação escleral de lente intra-ocular (LIO), implantes de lentes de câmara anterior para correção de afacia e ametropias altas, após aplicação de laser de argônio e ceratotomia radial. Outras etiologias de CB incluem distrofias endoteliais como a distrofia de Fuchs, tumores de câmara anterior como mixoma, alterações congênitas, como microcórnea e ice-síndrome, glaucomas agudo e neovascular e endotelite herpética ${ }^{(3,14-19)}$.

A ceratopatia bolhosa pode ocorrer em torno de 1 a $2 \%$ dos pacientes submetidos a cirurgia de catarata, o que representa cerca de 2 a 4 milhões de pacientes no mundo ${ }^{(1,20)}$.

Foi observado que após um procedimento cirúrgico intraocular ocorre diminuição do número e da capacidade de sítios da bomba de $\mathrm{Na}+, \mathrm{K}+$-ATPase endoteliais, localizados nas células basolaterais, responsáveis pela hidratação corneana e conseqüente sua transparência. Trabalhos recentes revelam a importância destes sítios também no epitélio corneano, que são afetados de forma subclínica após a cirurgia de catarata, podendo contribuir no desenvolvimento da ceratopatia bolhosa ${ }^{(1)}$.

Outra doença corneana que cursa com edema é a distrofia endotelial de Fuchs (DEF), a qual é clínica e histologicamente semelhante à ceratopatia bolhosa do pseudofácico, apesar de sua origem ser genética, com padrão hereditário autossômico dominante com penetrância incompleta. A histologia mostra espessamento da membrana de Descemet acompanhada de excrescências, conhecidas com "guttata". Com a idade, também ocorrem estas áreas semelhantes às guttatas, só que são periféricas. Nestas córneas, o aumento de disfunção endotelial e apoptose são fatores contribuintes para o edema corneano ${ }^{(1)}$.

Em córneas que apresentam o endotélio afetado por essas doenças, observa-se matriz extracelular alterada, alterações dos componentes da membrana basal, depósitos de fibrinogênio e roturas da membrana de Bowman, com eventual perda de glicosaminoglicanos no estroma ${ }^{(1,4)}$.

Estudos imunohistoquímicos mostram depósitos de componentes específicos da matriz extracelular, como tenascina-C e fibrilina-1, em pacientes com ceratopatia bolhosa.

A tenascina-C é uma glicoproteína que possui grande importância na cicatrização. Atua remodelando, reparando e no desenvolvimento ou formação de tecidos. Está normalmente ausente na córnea central, mas é encontrada na camada de colágeno posterior e áreas fibróticas subepiteliais de córneas com ceratopatia bolhosa. Aparece na lágrima de humanos após ceratectomia fototerapêutica (PTK) e em cicatrizes corneanas após muitos anos da incisão e em córneas inflamadas ${ }^{(21-22)}$.

A fibrilina-1 pertence à família de proteínas da matriz extracelular associada a microfibrilas elásticas ${ }^{(21)}$.

Nas córneas com ceratopatia bolhosa, há uma supra-regulação de fibrilina-1, que está depositada na face endotelial da membrana de Descemet e na camada de colágeno posterior. Também possuem metaloproteinase-2 no local da fibrose subepitelial e acúmulo de células inflamatórias, mas sem a participação de miofibroblastos ${ }^{(23)}$.

Estes não são requeridos para fibrose corneana, nem para remodelação e são raros na ceratopatia bolhosa ${ }^{(21)}$.

Sabe-se que fatores de crescimento e citocinas influenciam a proliferação e diferenciação celular, inflamação, cicatrização e fibrose. Foram encontrados níveis aumentados de interleucina-8 (IL-8), interleucina-2 (IL-2), fator de crescimento transformador (TGF- $\beta$ ), fator de crescimento similar à insulina-1 (IGF-1) e fator medular ósseo-4 (BMP-4) em córneas com ceratopatia bolhosa. O TGF- $\beta$ promove fibrose e depósitos na matriz extracelular, sem promover proteólise. Embora IGF-1 e TGF- $\beta$ s estejam aumentados na matriz extracelular corneana, eles são mais comumente encontrados nas áreas de fibrose de córneas com ceratopatia bolhosa. Estas interações entre os fatores de crescimento, matriz extracelular e metaloproteínas degradadoras da matriz são importantes, podendo ser um mecanismo para a perda de transparência corneana encontrada em córneas com ceratopatia bolhosa ${ }^{(2,21)}$.

A córnea absorve os raios ultravioletas (UV) que entram no olho e provavelmente é susceptível a danos de espécie oxigênio-reativa. Apresenta mecanismos de proteção, como a presença da ALDH3, que é uma isoenzima aldeído dehidrogenase que absorve os raios UV e remove aldeídos citotóxicos produzidos pela peroxidação lipídica causada pela radiação UV. A córnea é rica em enzimas antioxidantes, como superóxido dismutase, catalase, glutationa peroxidase, glutationa redutase, as quais removem radicais livres e espécies oxigênio-reativas geradas pelo $\mathrm{UV}^{(5)}$.

Em córneas com ceratopatia bolhosa foi observada a presença de malondialdeido, que é um bioproduto tóxico da peroxidação lipídica, no epitélio e principalmente em áreas com fibrose subepitelial e camadas posteriores. Em 2/3 das córneas com DEF foi encontrado o bioproduto no epitélio, mas este estava ausente nas camadas corneanas posteriores ${ }^{(5)}$.

\section{Tratamento}

O tratamento da $\mathrm{CB}$ pode ser feito clinicamente com agentes tópicos hipertônicos, antiinflamatórios, anti-hipertensivos, corticóides e lubrificantes ${ }^{(3)}$.

Alguns pacientes apresentam dor apesar do tratamento clínico e necessitam do uso de lentes de contato terapêuticas para melhora dos sintomas. Este tratamento deve ser considerado provisório devido ao risco de infecção ${ }^{(3-4)}$.

Nestes casos, deve-se pensar no tratamento cirúrgico como recobrimento conjuntival com técnica de Gunderson, a 
qual tem bons resultados, apesar de ser esteticamente limitada, podem acarretar ptose, e de comprometer a conjuntiva, sendo indesejável nos casos onde há necessidade de cirurgias para controle da pressão intra-ocular. Além disso piora o prognóstico do transplante de córnea devido à neovascularização corneana ${ }^{(3-4)}$. A ceratotomia anular promove, pela lesão do plexo nervoso corneano, a diminuição da sensiblidade e melhora da dor ${ }^{(4,24)}$.

A cauterização promove fibrose e vascularização com alteração da curvatura e anatomia da córnea causando aplanamento ${ }^{(3)}$.

As punções estromais anteriores, introduziriam projeções epiteliais ancorantes no estroma anterior o que não foi totalmente comprovado. Estudos imuno-histoquímicos demonstraram aumento da expressão de proteínas da matriz extracelular importantes na adesão das células epiteliais basais como fibronectina, laminina e colágeno tipo IV nos locais das punções estromais em olhos com ceratopatia bolhosa. A secreção desses componentes da membrana basal aumentaria a adesão epitelial no estroma subjacente, que está associada a formação de fibrose subepitelial, criando uma barreira à penetração de líquido no espaço subepitelial e diminuição da formação das bolhas subepiteliais. Possui custo baixo, sendo comum a necessidade de mais de uma sessão de punturas. Em cada sessão deve ser feito em torno de 100 punções por quadrante (400 punções), e as complicações são $\operatorname{raras}^{(3,25-27)}$.

A membrana amniótica tem sido utilizada para reconstrução de superfície ocular, pela sua capacidade de facilitar a epitelização, diminuir inflamação, vascularização e cicatrização, sendo utilizada no tratamento de defeito epitelial e dor em pacientes com ceratopatia bolhosa sintomáticos. Apresenta vantagens em relação à estética e não induz a neovavascularização. Existe a desvantagem do custo para preparo da membrana, e maior tempo cirúrgico ${ }^{(4,28-30)}$.

O PTK pode melhorar a dor por causar diminuição da espessura corneana. Esta diminuição ajudaria as células endoteliais remanescentes a manterem a hidratação da córnea. Também com a lesão do plexo corneano ocorre a diminuição da sensibilidade corneana. Além disso, a cicatrização da córnea induz um aumento de proteínas extracelulares como laminina, fibronectina, colágeno tipo IV e hemidesmossomos, os quais promovem maior adesão entre epitélio e estroma $^{(31-35)}$.

O mesmo raciocínio é usado para a ceratectomia lamelar automatizada, só que neste caso, a remoção de tecido corneano é feita com uso de microcerátomo tradicional, que retira um disco com $160 \mu$, substituindo a fotoablação com o "excimer laser”. É um procedimento rápido que usa anestesia tópica, fator importante, já que a maioria dos pacientes são idosos, os quais foram submetidos a vários procedimentos oculares, e apresentam dificuldade para a realização de procedimentos cirúrgicos que exijam decúbito dorsal por mais tempo.

O transplante de córnea é hoje o tratamento definitivo para melhora da acuidade visual e dor. Algumas limitações ocorrem, como a recuperação da acuidade visual, que pode ser lenta devido aos astigmatismos altos inerentes à técnica, principalmente no início do pós-operatório. Apesar da transparência dos enxertos ser boa, existe uma tendência maior em pacientes com CB e DEF de que ocorra migração das células endoteliais do enxerto para a córnea receptora, causando maior perda de células endoteliais. Por isso, está indicado um botão corneano de tamanho maior, mesmo que isto aumente os riscos de rejeição devido a maior proximidade ao limbo. Nestes casos, existe a necessidade eventual de cirurgias tríplices de fixação escleral ou iriana da LIO nos afácicos ou com LIOs descentradas, colocação de tubos para controle da pressão intra-ocular, reorganização do segmento anterior por terem antecedentes de cirurgias de cataratas complicadas. Isto faz com que o tempo cirúrgico e a reação inflamatória no pós-operatório seja maior, contribuindo para maior perda de células endoteliais e pior prognóstico visual ${ }^{(36-38)}$.

Tem-se avaliado a ceratoplastia lamelar posterior (DSEK e DLEK) para casos de doenças endoteliais da córnea. A técnica exige habilidade cirúrgica e dificulta qualquer atuação necessária na câmara anterior, porém apresenta a vantagem do menor risco de rejeição e da preservação da superfície do receptor. É uma técnica promissora mas deve-se observar maior número de casos e período de evolução, já que os primeiros estudos de contagem de células endoteliais após 2 anos de DSEK evidenciou perda celular maior do que nos transplantes penetrantes de córnea ${ }^{(39-40)}$.

\section{CONCLUSÃO}

Os estudos imuno-histoquímicos mostram linhas de pesquisa para se entender melhor a etiopatogênese da ceratopatia bolhosa, assim como as respostas da córnea aos tratamentos disponíveis. O transplante de córnea e o DSEK/DLEK são os tratamentos com melhores resultados na melhora da dor e da acuidade visual para os pacientes com potencial de visão. No caso de pacientes sem potencial visual ou que estejam aguardando o transplante de córnea ou DSEK/DLEK, o uso de tratamento clínico, uso de LC terapêutica e procedimentos mais invasivos como as punções estromais anteriores, transplante de membrana amniótica, PTK e ceratectomia lamelar automatizada, atenuam os sintomas de dor melhorando a qualidade de vida destes pacientes sintomáticos com CB.

\section{ABSTRACT}

Bullous keratopathy is characterized by corneal stromal edema with epithelial or subepithelial bullae due to cell loss and endothelial decompensation. In more advanced cases, subepithelial fibrosis, formation of a posterior collagenous layer or retrocorneal fibrous membrane, and corneal vascularization can occur. Decreased vision is present because the loss of corneal transparency with symptoms like tearing and pain caused by epithelial bullae that can rupture. Currently, bullous keratopathy is the most common indication for penetrating keratoplasty and regraft. The main etiology is the endo- 
thelial cell loss after ophthalmic surgeries as cataract surgery. Cataract affects approximately 20 million people worldwide, and this complication can occur in 1 to $2 \%$ of cataract surgeries. This study revised bullous keratopathy etiopathogenesis, clinical and surgical treatment available for this corneal disease.

Keywords: Corneal diseases/therapy; Corneal diseases/surgery; Corneal transplantation/etiology; Cataract extraction

\section{REFERÊNCIAS}

1. Ljubimov AV, Atilano SR, Garner MH, Maguen E, Nesburn AB, Kenney MC. Extracellular matrix and $\mathrm{Na}+, \mathrm{K}+-\mathrm{ATPase}$ in human corneas following cataract surgery: comparison with bullous keratopathy and Fuchs' dystrophy corneas. Cornea. 2002;21(1):74-80.

2. Spirin KS, Ljubimov AV, Castellon R, Wiedoeft O, Marano M, Sheppard D et al. Analysis of gene expression in human bullous keratopathy corneas containing limiting amounts of RNA. Invest Ophthalmol Vis Sci. 1999;40(13):3108-15.

3. Gomes JA, Haraguchi DZ, Zambrano DU, Villavicencio LI, Cunha MC, de Freitas D. Punções do estroma anterior no tratamento da ceratopatia bolhosa. Arq Bras Oftalmol. 2000;63(2):133-7.

4. Pires RT, Tseng SC, Prabhasawat P, Puangsricharern V, Maskin SL, Kim JC et al. Amniotic membrane transplantation for symptomatic bullous keratopathy. Arch Ophthalmol. 1999;117(10):1291-7.

5. Buddi R, Lin B, Atilano SR, Zorapapel NC, Kenney MC, Brown DJ. Evidence of oxidative stress in human corneal diseases. J Histochem Cytochem. 2002; 50(3):341-51.

6. Uchino Y, Goto E, Takano Y, Dogru M, Shinozaki N, Shimmura S et al. Long-standing bullous keratopathy is associated with peripheral conjunctivalization and limbal deficiency. Ophthalmology. 2006;113(7):1098-101.

7. Nguyen NX, Langenbucher A, Seitz B, Küchle M, Naumann GO. Impact of increased intraocular pressure on long-term corneal endothelial cell density after penetrating keratoplasty. Ophthalmologica. 2002;216(1):40-4.

8. Cosar CB, Sridhar MS, Cohen EJ, Held EL, Alvim Pde T, Rapuano CJ et al. Indications for penetrating keratoplasty and associated procedures, 1996-2000. Cornea. 2002;21(2):148-51.

9. Mkanganwi N, Nondo SI, Guramatunhu S. Indications for corneal grafting in Zimbabwe. Cent Afr J Med. 2000;46(11):300-2.

10. Maeno A, Naor J, Lee HM, Hunter WS, Rootman DS. Three decades of corneal transplantation: indications and patient characteristics. Cornea. 2000;19(1):7-11.

11. Dobbins KR, Price FW Jr, Whitson WE. Trends in the indications for penetrating keratoplasty in the midwestern United States. Cornea. 2000;19(6):813-6.

12. Calix Netto MJ, Giustina ED, Ramos GZ, Peccini RF, Sobrinho M, de Souza LB. Principais indicações de transplante penetrante de córnea em um serviço de referência no interior de São Paulo (Sorocaba-SP, Brasil). Arq Bras Oftalmol. 2006;69(5):661-4.

13. Chen WL, Hu FR, Wang IJ. Changing indications for penetrating keratoplasty in Taiwan from 1987 to 1999. Cornea. 2001;20(2):141-4.

14. Wollensak G, Green WR, Seiler T. Corneal myxoma. Jpn J Ophthalmol. 2002;46(2):193-7.

15. Kanellopoulos AJ. Penetrating keratoplasty and Artisan iris-fixated intraocular lens implantation in the management of aphakic bullous keratopathy. Cornea. 2004;23(3):220-4.

16. Pushker N, Tandon R, Vajpayee RB, Titiyal JS. Phakic-pseudophakic bullous keratopathy following implantation of a posterior chamber IOL in the anterior chamber to correct hypermetropia. Indian J Ophthalmol. 2000;48(3):235-6.

17. Takahashi H, Kashiwagi K, Kogure S, Tsukahara S. Bullous keratopathy after argon laser iridotomy presumably associated with latanoprost. Jpn J Ophthalmol. 2003;47(6):618-20.
18. Kawano H, Uesugi Y, Nakayasu K, Kanai A. Long-term follow-up for bullous keratopathy after sato-type anterior-posterior corneal refractive surgery. Am J Ophthalmol. 2003;136(6):1154-5.

19. Sugar A. Surgical trauma: pseudophakic and aphakic corneal edema. In: Krachmer JH. Cornea. St. Louis: Mosby; 2004. Chapter 112.

20. Kenney MC, Zorapapel N, Atilano S, Chwa M, Ljubimov A, Brown D. Insulin-like growth factor-I (IGF-I) and transforming growth factor-beta (TGFbeta) modulate tenascin- $\mathrm{C}$ and fibrillin-1 in bullous keratopathy stromal cells in vitro. Exp Eye Res. 2003;77(5):537-46.

21. Maseruka H, Ataullah SM, Zardi L, Tullo AB, Ridgway AE, Bonshek RE. Tenascin-cytotactin (TN-C) variants in pseudophakic/aphakic bullous keratopathy corneas. Eye. 1998;12(Pt 4):729-34.

22. Saghizadeh M, Chwa M, Aoki A, Lin B, Pirouzmanesh A, Brown DJ et al Altered expression of growth factors and cytokines in keratoconus, bullous keratopathy and diabetic human corneas. Exp Eye Res. 2001;73(2):179-89.

23. Akhtar S, Bron AJ, Hawksworth NR, Bonshek RE, Meek KM. Ultrastructura morphology and expression of proteoglycans, betaig-h3, tenascin-C, fibrillin-1, and fibronectin in bullous keratopathy. Br J Ophthalmol. 2001;85(6):720-31.

24. Koenig SB. Annular keratotomy for the treatment of painful bullous keratopathy. Am J Ophthalmol. 1996;121(1):93-4.

25. Sridhar MS, Vemuganti GK, Bansal AK, Rao GN. Anterior stromal puncture in bullous keratopathy: a clinicopathologic study. Cornea. 2001;20(6):573-9.

26. Gomes JA, Haraguchi DK, Zambrano DU, Izquierdo Júnior L, Cunha MC, de Freitas D. Anterior stromal puncture in the treatment of bullous keratopathy: six-month follow-up. Cornea. 2001;20(6):570-2.

27. Tsai TC, Su CY, Lin CP. Anterior stromal puncture for bullous keratopathy. Ophthalmic Surg Lasers Imaging. 2003;34(5):371-4.

28. Castellano AG, Bardal AM, Lago C, Moreira LB, Moreira H. Transplante de membrana amniótica na ceratopatia bolhosa. Arq Bras Oftalmol. 2004;67(6): $855-8$.

29. Mejía LF, Santamaría JP, Acosta C. Symptomatic management of postoperative bullous keratopathy with nonpreserved human amniotic membrane Cornea. 2002;21(4):342-5.

30. López Ferrando N, Celis Sánchez J, González Del Valle F, López Mondéjar E. [Monolayered amniotic membrane transplantation as a palliative treatment for bullous keratopathy] Arch Soc Esp Oftalmol. 2004;79(1):27-31.

31. Obeid WN, Richinho KP, Osores AP, Machado MA, Obeid RC, Vieira LA. Ceratectomia fototerapêutica (PTK) e ceratopatia bolhosa: relato de caso. Arq Bras Oftalmol. 2005;68(5):679-82.

32. Maini R, Sullivan L, Snibson GR, Taylor HR, Loughnan MS. A comparison of different depth ablations in the treatment of painful bullous keratopathy with phototherapeutic keratectomy. Br J Ophthalmol. 2001;85(8):912-5.

33. Lin PY, Wu CC, Lee SM. Combined phototherapeutic keratectomy and therapeutic contact lens for recurrent erosions in bullous keratopathy. Br J Ophthalmol. 2001;85(8):908-11.

34. Thomann U, Niesen U, Schipper I. Successful phototherapeutic keratectomy for recurrent erosions in bullous keratopathy. J Refract Surg. 1996;12(2): S290-2.

35. Lin PY, Wu CC, Lee SM. Combined phototherapeutic keratectomy and therapeutic contact lens for recurrent erosions in bullous keratopathy. $\mathrm{Br} \mathrm{J}$ Ophthalmol. 2001;85(8):908-11.

36. Langenbucher A, Seitz B, Nguyen NX, Naumann GO. Corneal endothelial cell loss after nonmechanical penetrating keratoplasty depends on diagnosis: a regression analysis. Graefes Arch Clin Exp Ophthalmol. 2002;240(5):387-92.

37. Langenbucher A, Nguyen NX, Seitz B. Predictive donor factors for chronic endothelial cell loss after nonmechanical penetrating keratoplasty in a regression model. Graefes Arch Clin Exp Ophthalmol. 2003;241(12):975-81.

38. Wearne MJ, Claoué C. Surgical management of coexisting pseudophakic bullous keratopathy and glaucoma. J Cataract Refract Surg. 1999;25(10):1317-20.

39. Busin M, Arffa RC, Sebastiani A. Endokeratoplasty as an alternative to penetrating keratoplasty for the surgical treatment of diseased endothelium: initial results. Ophthalmology. 2000;107(11):2077-82.

40. Melles GR, Lander F, Beekhuis WH, Remeijer L, Binder PS. Posterior lamellar keratoplasty for a case of pseudophakic bullous keratopathy. Am J Ophthalmol. 1999;127(3):340-1. 